
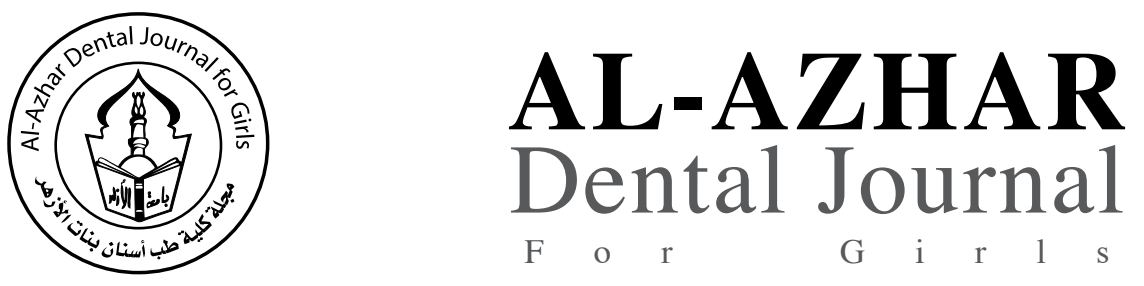

The Official Publication of The Faculty of Dental Medicine For Girls, Al-Azhar University Cairo, Egypt.

ADJ-for Grils, Vol. 4, No. 4, October (2017) - PP. 331:338

\title{
Podoplanin Protein Expression in Some Benign and Malignant Variants of Ameloblastoma (An immunohistochemical study)
}

\author{
Doaa A M. Habba ${ }^{(1)}$, Eman A. Abo Hager ${ }^{(2)}$ and Adel A. Shouman ${ }^{(2)}$
}

Codex : 39/1710

dentaljournal.forgirls@yahoo.com

\section{INTRODUCTION}

Odontogenic tumors represent a spectrum of lesions ranging from dental hamartomas and benign neoplasms to malignant, all arising from the odontogenic residues ${ }^{(1)}$. Ameloblastomas are benign locally aggressive, neoplasms of proliferating odontogenic epithelial origin, arising from one of the following: cell rests of enamel organ, remnants

\begin{abstract}
Aim: The aim of this study was to evaluate immunohistochemical expression of podoplanin among some benign and malignant variants of ameloblastoma. Material and methods: Thirty seven benign ameloblastoma $(\mathrm{AB})$ tumors were collected, (six cases of follicular $\mathrm{AB}$, three cases of acanthomatous $\mathrm{AB}$, four cases of granular $\mathrm{AB}$, three cases of basal cell $\mathrm{AB}$ and seven cases of plexiform pattern $\mathrm{AB}$ ), three cases of desmoplastic $\mathrm{AB}$, four cases of peripheral $\mathrm{AB}$ and seven cases of unicystic $\mathrm{AB}$ (three cases of luminal, two cases of intraluminal and two cases of mural) and eighteen malignant variants of ameloblastoma tumors were collected as paraffin embedded blocks. Immunohistochemical investigation using podoplanin antibody was done for all specimens. Results: The mean area percent of immunoexpression was greatest in malignant variants of ameloblastoma, followed by benign variants of ameloblastoma tumors and the least value recorded in acanthomatous ameloblastoma. The mean area percent of immunoexpression was greater in plexiform ameloblastoma in comparison to follicular ameloblastoma. The mean area percent of immunoexpression was greatest in ameloblastic carcinoma. Conclusions: Podoplanin is associated with the malignant transformation risk and is a potent biomarker for risk assessment in oral malignant transformation.
\end{abstract}

Podoplanin,

immunohistochemistry, ameloblastoma variants

\section{KEYWORDS}

1. Demonstrator of Oral and Dental Pathology, Faculty of Dental Medicine for Girls, Al Azhar University.

2. Professor of Oral and Dental Pathology, Faculty of Dental Medicine for Girls, Al Azhar University. 
of dental lamina, remenants of Hertwig's sheaths, developing enamel organ, basal cell epithelium of the jaws, epithelium of odontogenic cysts especially the dentigrous cyst, the odontomas and heterotrophic epithelium in other parts of the body particularly pituitary gland ${ }^{(2)}$.

Podoplanin is a type-1 transmembrane sialomucin-like glycoprotein, this protein has been largely used as an identifying marker of lymphatic endothelial cells due to its absence in blood endothelial cells and its expression is also considered to be associated with tooth development and tumor invasion ${ }^{(3,4)}$. podoplanin expression has been reported in other normal human tissues like podocytes, osteoblastic cells, osteocytes, basal keratinocytes, choroid plexus epithelial cells, myoepithelial cells, myofibroblasts of the prostate, follicular dendritic cells and alveolar type I cells and pathological conditions, such as squamous cell carcinoma of the lung, uterine cervix and head and neck, oral leukoplakia, pleomorphic adenoma and odontogenic tumors ${ }^{(5,6)}$.

Podoplanin mediates a pathway leading to collective and directional cell migration and forced expression of podoplanin lead to a dramatic change of cellular morphology. Also, adhesion and spreading of cells on the extracellular matrix protein fibronectin are enhanced by podoplanin expression. Induction of podoplanin expression results in multiple adjustments of intracellular signaling pathways and results in the modulation of RAS homolog gene family member A (Rho A) GTPase activities, the phosphorylation of ERM (Ezrin, Radixin, and Moesin) proteins and rearrangement of the actin cytoskeleton and enhances cell migration and invasion. Thus, podoplanin correlates with higher incidence of lymph node metastasis in early squamous cell carcinoma of the oral cavity and oropharyn $x^{(7,8)}$.

During the murine odontogenesis, preameloblasts exhibit a strong immunoreaction for podoplanin; however, once the pre-ameloblasts differentiate into secreting ameloblasts, podoplanin expression decreases drastically ${ }^{(9)}$. Several research studies have demonstrated that podoplanin seems to be expressed by aggressive tumors, with higher invasive and metastatic potential ${ }^{(10,11)}$. The relationship of PDPN in tumor invasion and its expression in human cancers indicate that it can be used as a biomarker for malignant transformation risk assessment and tumor progression biomarker for oral cancer ${ }^{(12)}$. Therefore, this study was conducted to evaluate immunohistochemical expression of podoplanin protein in some Benign and Malignant Variants of Ameloblastoma.

\section{MATERIALS AND METHODS}

\section{Case selection}

The specimens obtained for this study included benign and malignant ameloblastoma tumors collected as paraffin embedded blocks from the archives of Oral Pathology department of Alexandria University and Oral Pathology department of Asuite University. The specimens were divided into two groups, benign ameloblastoma tumors and malignant variants of ameloblastoma. Thirty seven benign ameloblastoma $(\mathrm{AB})$ tumors were collected, (six cases of follicular $\mathrm{AB}$, three cases of acanthomatous $\mathrm{AB}$, four cases of granular $\mathrm{AB}$, three cases of basal cell $\mathrm{AB}$ and seven cases of plexiform pattern $\mathrm{AB}$ ), three cases of desmoplastic $\mathrm{AB}$, four cases of peripheral $\mathrm{AB}$ and seven cases of unicystic $A B$ (three cases of luminal, two cases of intraluminal and two cases of mural). Eighteen malignant variants of ameloblastoma tumors were collected, ten cases of malignant ameloblastoma and eight cases of ameloblastic carcinoma.

\section{HISTOLOGICAL ANALYSIS}

Histological analysis with light microscope was performed on these specimens to verify diagnosis, and establish the histopathologic grading. 


\section{Immunohistochemical analysis}

Sections of $4 \mu \mathrm{m}$ thickness were mounted on electrically positive charged slides and deparaffinized by overnight incubation with xylene then, rehydrated in a gradual descending concentrations of ethanol followed by phosphate-buffered saline (PBS) wash. Blocking the endogenous peroxidase activity was performed by $3 \%$ hydrogen peroxide $\left(\mathrm{H}_{2} \mathrm{O}_{2}\right)$ for 5 minutes at room temperature. For antigen retrieval, tissue sections were put in glass jars containing $0.01 \mathrm{M}$ sodium citrate buffer ( $\mathrm{pH} 6.0$ ) and boiled in a microwave oven twice for 5 minutes each to enhance immunoreactivity (reserve the loss of antigenicity that occurred with some epitopes of formalinfixed paraffin-embedded tissues).The slides were allowed to cool and rinsed with PBS, pH 7.2. The immunohistochemical staining for podoplanin antibody was done according to the manufacturer's instructions using podoplanin mouse monoclonal antibody (Clone D2-40 Cat.No.IR072). The dilution used was 1:50 in phosphate buffered saline.

Detection was carried out using the universal kit (DAKO, Denmark) by washing slides in PBS for 5 minutes and incubated with secondary antibody that was biotinylated goat serum conjugated rabbit and mouse sera for 30 minutes. Sections were then washed for 5 minutes in PBS followed by development of antigen-antibody visualization by diaminobenzidine [DAB] in PBS containing $40 \% \mathrm{H}_{2} \mathrm{O}_{2}$. Sections were washed under running tap water for 10 minutes, then counterstained with Mayer's haematoxylin and mounted.

\section{Histomorphometric analysis}

Immunoreactivity, for Podoplanin; was evaluated by estimating the area percentage of positive immunostained cells in relation to the area examined in each field using Leica image analyzer computer system (Germany).The image analyzer consisted of a colored video camera, colored monitor, hard disc of hp personal computer connected to the microscope, and controlled by Leica Qwin 500 software.
The image analyzer was calibrated automatically to convert the measurement units (pixels) produced by the image analyzer program into actual micrometer units. The area and area percentage of podoplanin reactive areas were measured with reference to a standard measuring frame of area $11434.9 \mu \mathrm{m} 2$ using a magnification ( $\mathrm{x}$ 200). Using the color detection, reactive areas of positive immunostaining were masked by a blue binary color. Ten fields per each slide section of each patient were successively taken, to be histomorphometrically evaluated. Mean values were then obtained for each specimen.

\section{Statistical analysis}

Data were represented as means and standard deviation (SD) values, ANOVA test used to compare means of more than two groups. Tukey-Kramer multiple comparisons were used in the procedure of pair wise comparisons between the groups when ANOVA test is significant. The $\mathrm{P}$ value is significant if less than or equal 0.05 ( $\mathrm{P} \leq 0.05)$. Statistical analysis was performed by using instate graph pad version 3.10 and Microsoft® excel 2007.

\section{RESULTS}

\section{Immunohistochemical findings:}

The podoplanin immunostaining was detected positive cytoplasmic and membranous of basal cells while central cells are negative in follicular ameloblastoma. In acanthomatous ameloblastoma. Podoplanin immunostaining was seen in cytoplasm of the peripheral cells of the tumoral epithelial islands while it was negative in central cells both stellate reticulum like cells, squamous cells and keratin. Podoplanin was seen in cytoplasm and cell membrane of peripheral cells of the ameloblastoma islands. Some granular cells in the center showed membranous and faint cytoplasmic immunostaining, while other cells are negative of granular ameloblastoma (fig.1)

In basal cell ameloblastoma immunoexpression was seen in cell membrane and cytoplasm of both 
central and peripheral cells. While, podoplanin immunostaining was seen in cell membrane and cytoplasm of both of the ameloblast -like cells and few stellate reticulum- like cells of ameloblastoma strands of plexiform ameloblastoma. Podoplanin was seen in cytoplasm of the peripheral and central cells of ameloblastic epithelial islands of desmoplastic ameloblastoma. In malignant ameloblastoma podoplanin immunostaining was seen in the cell membrane and cytoplasm of both peripheral and central neoplastic cell (fig. 2).

In peripheral ameloblastoma showing cytoplasmic podoplanin immunostaining of basal cells of surface epithelium while spinous cells showed membranous staining. In ameloblastoma follicle, the central and peripheral cells revealed cytoplasmic and membranous podoplanin immunostaining. Podoplanin immunostaining was revealed in the cytoplasm of both the ameloblast-like cells (basal layer) and in the stellate reticulum-like cells (suprabasal layer) of the luminal unicystic ameloblastoma. In intra-luminal unicystic ameloblastoma showing podoplanin was cytoplasmic and membranous in both basal cell layer of the cyst lining and ameloblastic cord of the nodule that projecting into lumen. Few positive stellate reticulum like cells were revealed while the rest are negative. While, podoplanin immunostaining was seen in cytoplasm and cell membrane of the epithelial lining of the cyst and the cells of infiltrating follicles of ameloblastoma into the connective tissue wall of mural unicystic ameloblastoma.

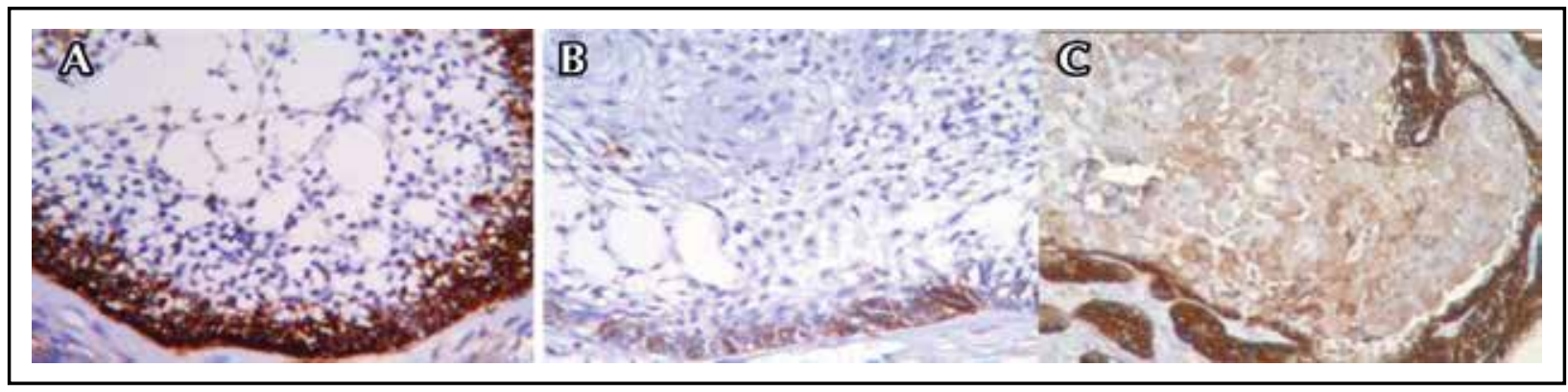

Fig. (1) Podoplanin immunostaining of cytoplasm and membranous of peripheral basal cell while central stellate reticulum cells are negative, A. Podoplanin immunostaning of cytoplasm of basal cell while stellate reticulum cells and squamous cells are negative,B. Podoplanin immunostaining of cytoplasm and membranous of basal cells while some granulare show immunostaining and others are negative, C, (A, B, C, podoplanin, X200).

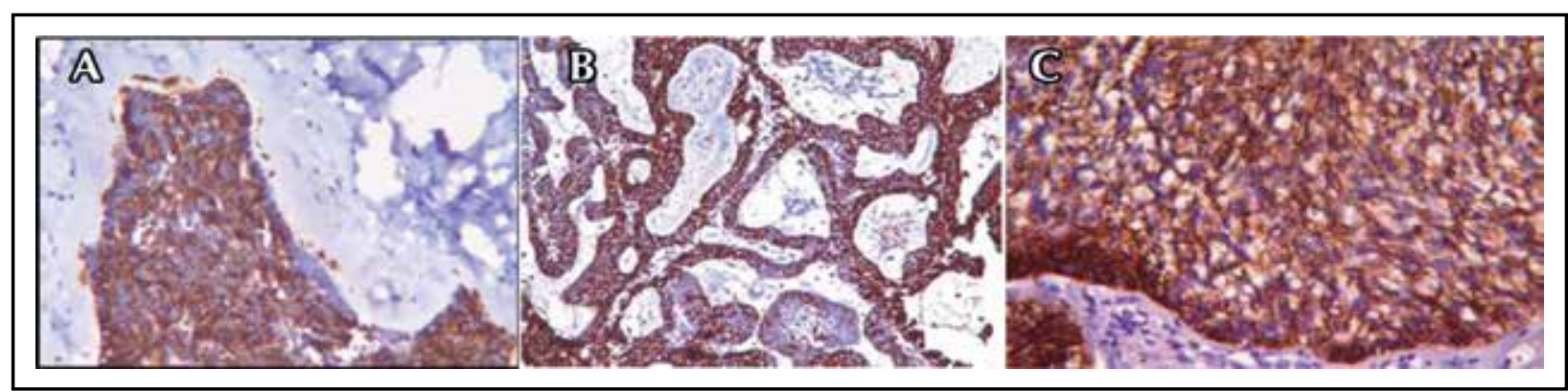

Fig. (2) Podoplanin immunostaining of cytoplasm of both peripheral cell and basaloid cell, A, Podoplanin immunostaining of cytoplasmic and membranous of peripheral cells and central reticulum stellatelike cell, B, Positive cytoplasmic and membranous podoplanin immunostaining of neoplastic cells of malignant ameloblastoma, C, (A, B, C, podoplanin, X200). 


\section{Statistical results}

The greatest mean area percent was recorded in ameloblastic carcinoma (63.101 \pm 9.76$)$, followed by malignant ameloblastoma (60.218 \pm 6.49$)$, whereas the lowest mean value was recorded in the acanthomatous ameloblastoma (6.761 \pm 1.68$)$. One way analysis of variance (ANOVA) test revealed a significant difference $(\mathrm{p}<0.0001)$. Tukey's post hoc test revealed a significant difference between both of the malignant variants and all other benign subtypes (table1, Fig.3).

Table (1) Values of area percent of immunoexpression in all benign and malignant variants and significance of the difference using ANOVA test.

\begin{tabular}{|l|l|l|l|l|l|}
\hline & Mean & Std Dev & Max & Min & P value \\
\hline Ab carcinoma & $63.101^{\mathrm{a}}$ & 9.763 & 78.451 & 49.234 & $<0.0001^{*}$ \\
\hline Malignant AB & $60.218^{\mathrm{a}}$ & 6.497 & 73.364 & 51.27 \\
\hline Plexiform AB & $33.031^{\mathrm{b}}$ & 4.498 & 40.711 & 27.118 \\
\hline Follicular AB & $29.66^{\mathrm{b}, \mathrm{c}}$ & 3.87 & 29.624 & 4.801 \\
\hline Basal AB & $29.442^{\mathrm{b}, \mathrm{d}}$ & 4.897 & 36.947 & 19.198 \\
\hline Peripheral AB & $28.455^{\mathrm{b}, \mathrm{d}}$ & 6.787 & 43.998 & 18.887 \\
\hline Unicystic AB & $24.834^{\mathrm{c}, \mathrm{d}, \mathrm{e}}$ & 7.431 & 44.326 & 16.288 \\
\hline Desmoplastic AB & $22.465^{\mathrm{c}, \mathrm{e}}$ & 6.922 & 36.775 & 11.248 \\
\hline Granular AB & $8.807^{\mathrm{f}}$ & 2.85 & 49.618 & 18.482 \\
\hline Acanthomatous AB & $6.761^{\mathrm{f}}$ & 1.68 & 9.502 & 4.815 \\
\hline
\end{tabular}

*significant at $p<0.05 \quad$ Tukey's post hoc test: means with different superscript letters are significantly different.

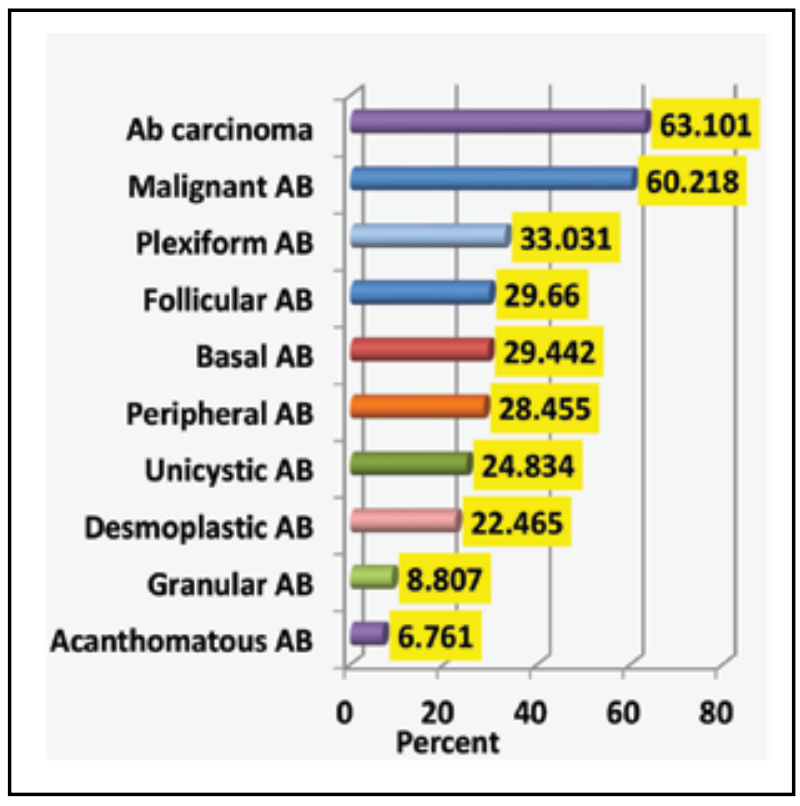

Fig. (3) Bar chart showing means area of immunoexpression in all benign and malignant variants of ameloblastoma.

\section{DISCUSSION}

In the present study, 55 cases of ameloblastoma representing the different histological types including follicular, plexiform, demoplastic, peripheral, unicystic, malignant $\mathrm{AB}$ and ameloblastic carcinoma were investigated in order to study the pattern and degree of podoplanin immunoreactions. All ameloblastoma variants showed positive immunoreactions for podoplanin with variable expression. These results are in accordance with previous studies they stated that all ameloblastoma variants displayed membranous and/or cytoplasmic expression of podoplanin and its expression were consistent with the cell type and tumor location. Moreover, they revealed that the epithelial tumoral cells in intimate contact with the stroma were 
stained strongly positive for podoplanin, while the central cells of the islands were negative ${ }^{(13,14)}$.

In the present study, the distributions of positive immune reaction for podoplanin were variable among the different tumors and in the various cases of the same tumors. The podoplanin expression revealed that malignant variants had higher significant difference than benign variants $(\mathrm{p}<0.0001)$ where ameloblastic carcinoma showed the statistically significant highest value mean (63.101 \pm 9.76$)$ while, plexiform variant of $A B$ showed statistically significant highest value among the benign variants (33.031 \pm 4.498$)$ while, acanthomatous AB showed statistically significant value $(6.761 \pm 1.68)$. These results were in accordance with previous studies where podoplanin is associated with the malignant transformation risk and is apotent biomarker for risk assessment in oral malignant transformation ${ }^{(15,16)}$.

In the present study, a strong positivity for podoplanin was observed on the cell membrane, at cell-cell boundaries, where there was an intimate contact with stroma and in the cytoplasm of peripheral columnar cells of ameloblastoma. Whereas, negative podoplanin reaction was detected in the central stellate reticulum-like cells. These results were in position with results of many studies ${ }^{(13,14,17)}$.

In the presnt study, follicular ameloblastomas showed positive membranous and cytoplasmic immunostaining $(83.3 \%)$ of podoplanin in the outer columnar cells of epithelial islands but central stellate reticulum cells were negative. Similary, it was found that podoplanin immunoprofile in ameloblastoma is consistently typified by strong podoplanin expression in pre- ameloblast-like cells and low immunoreactivity for podoplanin in stellate reticulum-like cells, that provide the evidence of a relationship between podoplanin and odontogenic cellular activity (i.e. the protein is expressed during intense proliferative activity in odontogenic cells and when these cells reach maturity or a stable state, there is a reduction or lack of podoplanin immunoreactivity). So, the odontogenic tumoral cells with low mitotic activity, such as the central stellate reticulum-like cells, keratinized acanthomatous cells did not express podoplanin ${ }^{(18)}$.

Regarding acanthomatous ameloblastoma, podoplanin positive immunostaining was seen in $(66.6 \%)$ of cases, and has significantly lowest value of expression compared to all benign variants of $\mathrm{AB}$ (6.76). The acanthomatous variants in the present cases demonstrate positive podoplanin immunostaining of cytoplasm in a focal pattern of the peripheral cells of the tumoral epithelial islands and negative for central stellate reticulum like cells and acanthomatous cells. These results were in accordance other the study that revealed podoplanin-positive cells were observed in the peripheral layer of tumor nests, but were absent in keratinized areas of the acanthomatous AB subtype, probably because of terminal differentiation of the tumor cells resulting from maturation and/or degenerative changes. Based on these concepts, podoplanin may be a useful marker of cells with the capacity for further maturation ${ }^{(19)}$.

In the current study, podoplanin positive immunostaining was seen in $(50 \%)$ of granular cell ameloblastoma cases. The expression was significantly the lowest mean value of all benign variants of $\mathrm{AB}$ (8.80). Positive podoplanin immunostaining was found in cytoplasm and cell membrane of peripheral cells of the ameloblastoma islands and some granular cells showed faint immunostaining and others were negative. These results in agree with previous studies, where granular cells of those tumours present weak or negative podoplanin expression as granular cells are more mature, less active and more stable ${ }^{(13,17)}$.

In the current study, plexiform ameloblastma showed membranous and cytoplasmic immunostaining in peripheral epithelial cuboidal and central cells. This result agreed with that of previous studies where plexiform ameloblastoma exhibiting podoplanin expression in peripheral and central 
cells, that plexiform $\mathrm{AB}$ resembles the tooth germ in the dental lamina stage, when the differentiation process of the odontogenic epithelium has not initiated. This lack of cellular differentiation may reflect the homogeneity of podoplanin expression that mean cells of plexiform $\mathrm{AB}$ are immature cells and proliferative cells. This may also explain the $100 \%$ positivity of plexiform $A B$ in the current study ${ }^{(20,17)}$.

Regarding malignant ameloblastoma and ameloblastic carcinoma showed positive immunostaining in $(100 \%)$ of cases, respectively. There was no significant difference between both malignant variants $(P=0.447)$. These results were in accordance with other studies that revealed enhanced podoplanin expression along the invasive fronts of malignant $A B$, indicating that the invasive front is composed of the most aggressive cells and is a region of active molecular interactions that may potentially affect tumor progression that podoplanin might be a potential prognostic indicator of tumor aggressiveness in malignant tumors ${ }^{(21)}$.

In the present sudy, increased mean value of PDPN immunoexpression of ameloblastic carcinoma than that of malignant ameloblastoma although indicate the association between cellular atypia and dysplasia with increased PDPN expression which consequently leads tumor aggressiveness. These results were in agreement with many authers who studied the role of PDPN in carcinomas of different organs ${ }^{(22)}$. The discussion about PDPN and its participation in odontogenic tumours is interesting topic of study. The current study supports the presence of significant role for PDPN in development and progression of ameloblastoma.

\section{CONCLUSIONS}

Podoplanin is associated with invasive behavior of ameloblastoma as well as progression from benign to malignant ameloblastoma. Depending on variable expression of PDPN in different ameloblastoma subtypes, we consider this marker as a reliable one to detect the highly invasive and aggressive tumors (malignant variants, plexiform and follicular) compared to less aggressive variants (acanthomatous and granular). Podoplanin can be used as a biomarker for detection of odontogenic tumorgenesis and for advanced stage of malignant transformation of ameloblastoma.

\section{REFERENCES}

1. Schrohl S, Anderson H, Sweep F, Schmitt M, Harbeck N, Foekens J. Tumor markers: From laboratory to clinical utility. Mol Cell Proteomics. 2013; 2(6):378-87.

2. Hennry M, Oherric S. Odontogenic tumors of the jaw in oral and maxillofacial surgery. $2^{\text {nd }}$ ed. ALTBS, New Delhi, India. 2009; 626-63.

3. Friedrich R, Zustin J. Calcifying epithelial odontogenic tumour of the maxilla: a case report with respect to immunohistochemical findings. In Vivo. 2011; 25:259-64.

4. Kikuchi K, Ito S, Inoue H, Gonzalez-Alva P, Miyazaki Y, Sakashita H. Immunohistochemical expression of podoplanin in so-called hard a-keratin-expressing tumors, including calcifying cystic odontogenic tumor, craniopharyngioma, and pilomatrixoma. J Oral Sci. 2012; 54(2): 165-75.

5. Kreppel M, Scheer M, Drebber U, Ritter L, Zoller J. Impact of podoplanin expression in oral squamous cell carcinoma: clinical and histopathologic correlations. Virchows Arch. 2010; 456:473-82.

6. Friedrich R, Scheuer H, Zustin J. Expression of podoplanin in nevoid basal cell carcinoma syndrome-associated keratocystic odontogenic tumours. Anticancer Res. 2012; 32:2125-7.

7. Huber G, Fritzsche R, Zullig L, Storz M, Graf N, Haerle K, Jochum W, Stoeckli J, Moch H. Podoplanin expression correlates with sentinel lymph node metastasis in early squamous cell carcinomas of the oral cavity and oropharynx. Int J Cancer. 2011; 129(6): 1404-9.

8. De Vicente C, Rodrigo $\mathrm{P}$, Rodriguez-Santamarta $\mathrm{T}$, Lequerica-Fernandez P, Allonca E, García-Pedrero $\mathrm{M}$. Podoplanin expression in oral leukoplakia: tumorigenic role. Oral Oncol. 2013; 49(6): 598-603.

9. Imaizumi Y, Amano I, Tsuruga E, Kojima H, Sawa Y. Immunohistochemical examination for the distribution of podoplanin-expressing cells in developing mouse molar tooth germs. Acta Histochem Cytochem. 2010; 43: 115-21. 
10. Toll A, Gimeno-Beltran J, Ferrandiz-Pulido C, Masferrer E, Yebenes M, Jucgla A, Abal L, Marti R, Sanmartin O, Baro T, Casado B, et al. D2-40 immunohistochemical overexpression in cutaneous squamous cell carcinomas: a marker of metastatic risk. J Am Acad Dermatol. 2012; 67(6): 1310-8.

11. Ohta M, Abe A, Ohno F, Hasegawa Y, Tanaka H, Maseki S, Kondo E, Kurita K, Nakanishi H. Positive and negative regulation of podoplanin expression by TGF- $\beta$ and histone deacetylase inhibitors in oral and pharyngeal squamous cell carcinoma cell lines. Oral Oncol. 2013; 49(1):20-6.

12. Rodrigo P, Garcia-Carracedo D, Gonzalez V, Mancebo G, Fresno F, Garcia- Pedrero J. Podoplanin expression in the development and progression of laryngeal squamous cell carcinomas. Mol Cancer. 2010; 9:48-57.

13. Zustin J, Scheuer H, Friedrich R. Podoplanin expression in human tooth germ tissues and cystic odontogenic lesions: an immunohistochemical study. J Oral Pathol Med. 2010; 39:115-20.

14. Gonzalez-Alva P, Tanaka A, Oku Y, Miyazaki Y, Okamoto E, Fujinami M, et al.

15. Enhanced expression of podoplanin in ameloblastomas. J oral Pathol Med. 2010; 39:10-9.

16. Kawaguchi H, El-Naggar A, Papadimitrakopoulou V, Ren H, Fan Y, Feng L, et al. Podoplanin: a novel marker for oral cancer risk in patients with oral premalignancy. J Clin Oncol. 2008; 26: 354-60.
17. Shi P, Liu W, Zhou Z, He Q, Jiang W. Podoplanin and ABCG2: malignant transformation risk markers for oral lichen planus. Cancer Epidemiol Biomarkers Prev. 2010; 19: 844-9.

18. Tjioe KC, Oliveira DT, Soares CT, Lauris JR, Damante JH. Is podoplanin expression associated with the proliferative activity of ameloblastomas? Oral Dis. 2012; 18:673-6.

19. Caetano Ados S, Tjioe K, Faustino S, Hanemann J, Belone Ade F, Soares T, Oliveira D. Immunolocalization of podoplanin in benign odontogenic tumours with and without ectomesenchyme. Arch Oral Biol. 2013; 58:408-15.

20. Ito T, Ishii G, Nagai K, Kojika M, Murata Y, Atsumi N, Nishiwaki Y, Miyazaki E, Kumamoto T, Ochiai A. Low podoplanin expression of tumor cells predicts poor prognosis in pathological stage IB squamous cell carcinoma of the lung, tissue microarray analysis of 136 patients using 24 antibodies. Lung Cancer. 2009; 63: 418-24.

21. Gonzalez-Alva P, Tanaka A, Oku Y, Okamoto E, Fujinami M, Yoshida N, Kikuchi K, Ide F, Sakashita H, Kusama K. Enhanced expression of podoplanin in ameloblastomas. J Oral Pathol Med. 2009; 39: 103-6.

22. Wang A, Zhang B, Huang H, Zeng D, Tao Q, Wang J, Pan C. Suppression of localinvasion of ameloblastoma by inhibition of matrix metalloproteinase-2in vitro. BMC Cancer. 2008; 8: 182.

23. Wicki A, Lehembre F, Wick N, Hantusch B, Kerjaschki D, Christofori G. Tumor invasion in the absence of epithelialmesenchymal transition: podoplanin-mediated remodeling of the actin cytoskeleton. Cancer Cell. 2006; 9:261-72. 